\title{
AGE CLASSIFICATION WITH SHAPE PATTERNS DERIVED FROM CENTRAL PIXEL FLOODING MATRIX ON FACIAL IMAGES
}

\author{
P.Chandra Sekhar Reddy ${ }^{1}$ and Bhanu Sreekar Reddy Karumuri ${ }^{2}$
}

\begin{abstract}
Humans can easily categorize persons into different age groups from facial images of people. The Age classification based on computer vision has widespread applications. For automatic age classification, in this paper shape patterns on Central Pixel Flooding Matrix (CPFM) are used to classify persons with face images into two classes, child and adult. The CPFM forms a textured image over the facial image by considering neighborhood pixels which have the same intensity as a central pixel. The shape patterns Lower Triangular Matrix Pattern (LTMP), Upper Triangular Matrix Pattern (UTMP) and Tri-Diagonal Matrix Pattern (TDMP) on CPFM of facial images are calculated and these features are used for age classification. The experimental results on the FGNet aging database have shown that this method is more efficient compared to other methods for age grouping of facial images.

Keywords: Age classification, Central Pixel Flooding Matrix, LTMP, UTMP, and SSP.
\end{abstract}

\section{INTRODUCTION}

Age estimation is an important in computer vision applications like forensic and criminal investigations, the determination of retirement age, military age, access to web services with age criteria, supervision of minors, demographics, commercial adds and so on. The Age estimation with texture feature [1, 2], contour features and texture features separately [3, 4]. An extraction of skin feature for automatic skin aging estimation [5]. Age classification methods are categorized into three categories [6]. They are an anthropometric model [3,7], aging pattern subspace[8], and age regression[9-12] categories. Recently facial emotion algorithms based on spectral features in ECG signals [13], LBP models [14] are developed. Recently various methods for age classification and age grouping are developed by Vijaya Kumar et. al.[15], Jangala Sasi Kiran et. al.[16] and Chandra Sekhar Reddy et. al.[17]. To address this problem, this paper focuses on the grouping of facial images into two classes, child and adult. The present paper considers the CPFM of the facial image as a texture. So shape pattern based texture classification method is proposed on the CPFM of facial images. The rest of the paper is organized as follows. In section2, proposed methodology adopted, Experimental results and discussions are given in section3 and conclusion is drawn in section4.

\section{METHODOLOGY}

In Face recognition and age classification applications facial image is considered as texture. To evaluate micro texture features of the face, this paper uses the shape patterns over central pixel flooding matrix computed on facial images for age classification. The central pixel flooding matrix forms a group of pixels which have the same intensity value as the central pixel over the $3 \times 3$ neighborhood. The central pixel flooding, computed on the image gathers textural information. The

\footnotetext{
${ }^{1}$ Professor, CSE Dept., Gokaraju Rangaraju Institute of Engineering and Technology,Hyd., pchandureddy@yahoo.com

${ }^{2}$ B.Tech Final year Student, CSE Dept., Gokaraju Rangaraju Institute of Engineering and Technology, Hyd. bhanusreekaredu@gmail.com
} 
set of shape patterns lower triangular matrix pattern, upper triangular matrix pattern and tri-diagonal matrix pattern on this CPFM are computed.

The frequency occurrences of these features are evaluated. The different aged people facial images can have a variation of skin texture or wrinkles, this can be significantly identified with shape patterns. The proposed method for age classification consists of three steps as shown in the block diagram of Fig.1.

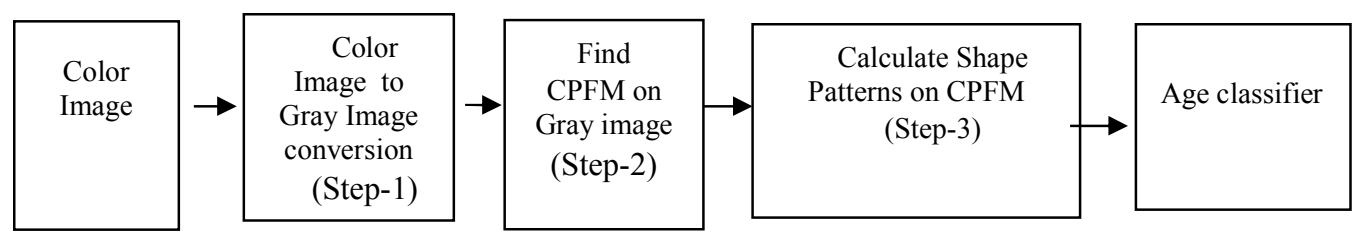

Fig.1: Age classification scheme with Shape patterns on central pixel flooding matrix of images.

\section{A. Color image to Gray Image conversion}

The original colour image is converted into a grey level image using RGB color quantization method.

\section{B. Computing Central Pixel Flooding Matrix (CPFM)}

The central pixel flooding forms a group of pixels which have the same intensity value as the central pixel over the $3 \times 3$ neighborhood. In the $3 \times 3$ neighborhood, for all the neighbors which have the same intensity value as the center pixel, then these values are kept unchanged otherwise, it is set to zero. The $3 \times 3$ block obtained from this process is called a central pixel flooding. The CPFM is computed over the whole image is described as follows.

(1) Central pixel floodings I1 $(x, y), I 2(x, y), I 3(x, y)$ and $I 4(x, y)$ are computed starting from pixel $(1,1),(1,2),(2,1)$ and $(2,2)$ respectively with $3 \times 3$ block from left-to-right and top-to-bottom throughout image $I(m, n)$ with a step-length of three pixels along both horizontal and vertical directions.

(2) The final central pixel flooding matrix, denoted by $\operatorname{CPFM}(x, y)$ is computed using equation 1.

$\operatorname{CPFM}(\mathrm{x}, \mathrm{y})=\mathrm{p}$

Where $\mathrm{p}$ is avg (or) avg_g $\operatorname{avg}=(\mathrm{I} 1(\mathrm{x}, \mathrm{y})+\mathrm{I} 2(\mathrm{x}, \mathrm{y})+\mathrm{I} 3(\mathrm{x}, \mathrm{y})+\mathrm{I} 4(\mathrm{x}, \mathrm{y})) / \mathrm{r}, \mathrm{r}$ is the number of non-zero intensity values .

avg_g $=$ a value which is just greater than avg and equal to one of four intensity values I1(x,y), $\mathrm{I} 2(\mathrm{x}, \mathrm{y}), \mathrm{I} 3(\mathrm{x}, \mathrm{y})$ and $\mathrm{I} 4(\mathrm{x}, \mathrm{y})$ at position $(\mathrm{x}, \mathrm{y})$.

An example of central pixel flooding matrix detection is shown in Fig.2. 


\begin{tabular}{|c|c|c|}
\hline 8 & 7 & 8 \\
\hline 8 & 8 & 4 \\
\hline 5 & 8 & 9 \\
\hline
\end{tabular}

(a) $3 \times 3$ block with gray values

\begin{tabular}{|c|c|c|}
\hline 8 & 0 & 8 \\
\hline 8 & 8 & 0 \\
\hline 0 & 8 & 0 \\
\hline
\end{tabular}

(b) Central pixel flood

\begin{tabular}{|l|l|l|l|l|l|l|}
\hline 5 & 5 & 7 & 9 & 2 & 3 & 8 \\
\hline 4 & 6 & 9 & 1 & 8 & 8 & 8 \\
\hline 3 & 3 & 6 & 1 & 3 & 4 & 4 \\
\hline 8 & 5 & 5 & 3 & 1 & 6 & 4 \\
\hline 5 & 8 & 9 & 5 & 1 & 4 & 4 \\
\hline 4 & 8 & 9 & 9 & 5 & 4 & 6 \\
\hline 8 & 9 & 5 & 3 & 5 & 4 & 4 \\
\hline
\end{tabular}

(c ) 7x7 Image block

\begin{tabular}{|l|l|l|l|l|l|l|}
\hline 0 & 0 & 0 & 9 & 0 & 0 & 8 \\
\hline 0 & 0 & 9 & 0 & 8 & 8 & 8 \\
\hline 0 & 0 & 0 & 0 & 0 & 0 & 0 \\
\hline 0 & 0 & 0 & 0 & 1 & 0 & 4 \\
\hline 0 & 0 & 9 & 0 & 1 & 4 & 4 \\
\hline 0 & 0 & 9 & 9 & 0 & 4 & 0 \\
\hline 0 & 0 & 0 & 0 & 0 & 0 & 0 \\
\hline
\end{tabular}

(e) $I 2(x, y)$

\begin{tabular}{|l|l|l|l|l|l|l|}
\hline 0 & 0 & 0 & 0 & 0 & 0 & 0 \\
\hline 0 & 6 & 0 & 0 & 0 & 0 & 0 \\
\hline 0 & 0 & 6 & 0 & 0 & 4 & 4 \\
\hline 0 & 0 & 0 & 0 & 0 & 0 & 4 \\
\hline 0 & 0 & 9 & 0 & 0 & 4 & 4 \\
\hline 0 & 0 & 9 & 9 & 0 & 4 & 0 \\
\hline 0 & 0 & 0 & 0 & 0 & 4 & 4 \\
\hline
\end{tabular}

(g) $I 4(x, y)$

\begin{tabular}{|l|l|l|l|l|l|l|}
\hline 0 & 0 & 0 & 0 & 0 & 0 & 0 \\
\hline 0 & 6 & 0 & 0 & 8 & 8 & 0 \\
\hline 0 & 0 & 6 & 0 & 0 & 0 & 0 \\
\hline 8 & 0 & 0 & 0 & 1 & 0 & 0 \\
\hline 0 & 8 & 0 & 0 & 1 & 0 & 0 \\
\hline 0 & 8 & 0 & 0 & 0 & 0 & 0 \\
\hline 0 & 0 & 0 & 0 & 0 & 0 & 0 \\
\hline
\end{tabular}

(d) $\mathrm{I1}(\mathrm{x}, \mathrm{y})$

\begin{tabular}{|l|l|l|l|l|l|l|}
\hline 0 & 0 & 0 & 0 & 0 & 0 & 0 \\
\hline 0 & 0 & 0 & 0 & 0 & 0 & 0 \\
\hline 3 & 3 & 0 & 0 & 3 & 0 & 0 \\
\hline 0 & 0 & 0 & 3 & 0 & 0 & 0 \\
\hline 0 & 8 & 0 & 5 & 0 & 0 & 0 \\
\hline 0 & 8 & 0 & 0 & 5 & 0 & 0 \\
\hline 8 & 0 & 0 & 0 & 5 & 0 & 0 \\
\hline
\end{tabular}

(f) $\mathrm{I} 3(\mathrm{x}, \mathrm{y})$

\begin{tabular}{|l|l|l|l|l|l|l|}
\hline 0 & 0 & 0 & 9 & 0 & 0 & 8 \\
\hline 0 & 6 & 9 & 0 & 8 & 8 & 0 \\
\hline 3 & 3 & 6 & 0 & 0 & 4 & 4 \\
\hline 8 & 0 & 0 & 3 & 1 & 0 & 4 \\
\hline 0 & 8 & 9 & 5 & 1 & 4 & 4 \\
\hline 0 & 8 & 9 & 9 & 5 & 4 & 0 \\
\hline 8 & 0 & 0 & 0 & 5 & 4 & 4 \\
\hline
\end{tabular}

(h) Central pixel flood matrix

Fig.2. Detection of Central pixel flood matrix 


\section{Evaluation of Shape Patterns}

The shape patterns on $3 \times 3$ block are defined as follows. In lower triangular matrix pattern, nonzero elements occur on principal diagonal and below this diagonal. In upper triangular matrix pattern, nonzero elements occur on principal diagonal and above this diagonal. In tri diagonal matrix pattern, nonzero elements occur on principal diagonal, below and above this diagonal. These patterns are shown in Fig.3. The frequency occurrences of these shape patterns are computed on CPFM and sum of these shape patterns are computed.

\begin{tabular}{|l|l|l|}
\hline $\mathrm{N}$ & 0 & 0 \\
\hline $\mathrm{N}$ & $\mathrm{N}$ & 0 \\
\hline $\mathrm{N}$ & $\mathrm{N}$ & $\mathrm{N}$ \\
\hline
\end{tabular}

(a)

\begin{tabular}{|l|l|l|}
\hline $\mathrm{N}$ & $\mathrm{N}$ & $\mathrm{N}$ \\
\hline 0 & $\mathrm{~N}$ & $\mathrm{~N}$ \\
\hline 0 & 0 & $\mathrm{~N}$ \\
\hline
\end{tabular}

(b)

\begin{tabular}{|l|l|l|}
\hline $\mathrm{N}$ & $\mathrm{N}$ & 0 \\
\hline $\mathrm{N}$ & $\mathrm{N}$ & $\mathrm{N}$ \\
\hline 0 & $\mathrm{~N}$ & $\mathrm{~N}$ \\
\hline
\end{tabular}

(c)

Fig 3: a) Lower Triangular Matrix Pattern (LTMP) b) Upper Triangular Matrix Pattern (UTMP) c) Tri Diagonal Matrix Pattern(TDMP), where $\mathrm{N}$ is a nonzero element

\section{RESULTS AND DISCUSSIONS}

The proposed method established a database of the 1002 face images collected from FG-NET database and other 600 images collected from the scanned photographs and sample of these images are shown in Fig.4. In this method, images are classified into two groups as a child (upto 18 years) and adult (above 18 years) based on frequency occurrences of shape patterns. The frequency occurrence of each shape patterns i.e. FLTMP, FUTMP, and FTDMP on CPFM and Sum of Shape Patterns (SSP) are evaluated on facial images and the results for a sample of 20 images is listed out in table1.

From the table1 it is observed that FLTMP and FUTMP are dominant patterns and FTDMP patterns are not dominant patterns as these are zero for age varying images. So FLTMP, FUTMP, and SSP can be considered for age classification. The algorithm1 is proposed to classify images into two categories adult and child with shape patterns FLTMP, FUTMP, and SSP.

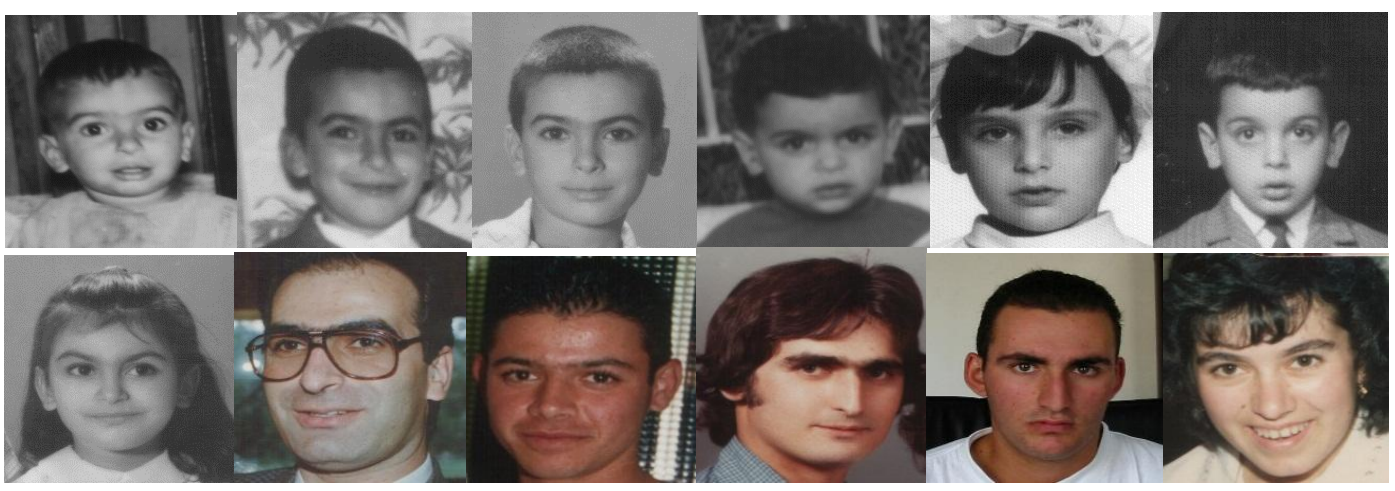

Fig 4: Sample images from FG-NET Database 
Table 1. The frequency of occurrences of LTMP, UTMP, TDMP and SSP

\begin{tabular}{|l|l|l|l|l|}
\hline IMAGE & FLTMP & FUTMP & FTDMP & SSP \\
\hline $001 \mathrm{~A} 02$ & 8357 & 13524 & 0 & 21881 \\
\hline $001 \mathrm{~A} 05$ & 11584 & 19370 & 0 & 30954 \\
\hline $001 \mathrm{~A} 08$ & 14996 & 22115 & 0 & 37111 \\
\hline $011 \mathrm{~A} 02$ & 8477 & 14581 & 0 & 23058 \\
\hline $002 \mathrm{~A} 04$ & 15932 & 21713 & 0 & 37645 \\
\hline $002 \mathrm{~A} 12$ & 15442 & 22186 & 0 & 37628 \\
\hline $011 \mathrm{~A} 07$ & 14697 & 18329 & 0 & 33026 \\
\hline $008 \mathrm{~A} 06$ & 15583 & 21534 & 0 & 37117 \\
\hline $008 \mathrm{~A} 08$ & 13820 & 17424 & 0 & 31244 \\
\hline $012 \mathrm{~A} 04$ & 12590 & 14312 & 0 & 26902 \\
\hline $001 \mathrm{~A} 33$ & 24295 & 31142 & 0 & 55437 \\
\hline $037 \mathrm{~A} 19$ & 18161 & 25722 & 0 & 43883 \\
\hline $011 \mathrm{~A} 40$ & 28104 & 35112 & 0 & 63216 \\
\hline $013 \mathrm{~A} 25$ & 18843 & 25035 & 0 & 43878 \\
\hline $013 \mathrm{~A} 34$ & 19014 & 22398 & 0 & 41412 \\
\hline $016 \mathrm{~A} 19$ & 19440 & 23550 & 0 & 42990 \\
\hline $025 \mathrm{~A} 22$ & 18326 & 23756 & 0 & 42082 \\
\hline $025 \mathrm{~A} 39$ & 24053 & 25341 & 0 & 49394 \\
\hline $027 \mathrm{~A} 20$ & 19362 & 24395 & 0 & 43757 \\
\hline $027 \mathrm{~A} 41$ & 18019 & 25392 & 0 & 43411 \\
\hline $030 \mathrm{~A} 26$ & 18128 & 25622 & 0 & 50540 \\
\hline $035 \mathrm{~A} 21$ & 22049 & 28491 & 0 & \\
\hline & & & & 0 \\
\hline
\end{tabular}

Algorithm 1: Age classification using frequency occurrences of LTMP, UTMP, TDMP and SSP patterns on CPFM

Let FLTMP, FUTMP, and FTDMP be frequency occurrences of LTMP, UTMP and TDMP patterns and SSP is the sum of frequency occurrences shape patterns.

Begin

if $(($ FLTMP $<16000) \&(\mathrm{UTMP}<22200)$

$\&(\mathrm{SSP}<38000))$

write ("Image is child")

else

write ("Image is adult")

end

The algorithm1 classified the FG-Net facial images into two groups with $98 \%$ correct classification rate. The proposed method for age grouping is compared with the existing methods, Age 
classification with shape features on lbp based texton by P Chandra Sekhar Reddy et.al.[18]. Child and adulthood classification with geometrical features by Chandra Mohan et.al.[19] and other age classification methods Young H.Kwon et.al.[3] , Tsuneo Kanno et. al.[20] and Wen-Bing Horng Cheng et.al.[4]. The comparison table for proposed and existing methods with the percentage of classification rate is listed in table2. The results indicate that the proposed scheme outperforms with other methods.

Table 2: Comparison of the proposed Shape patterns on CPFM with other methods.

\begin{tabular}{|c|c|c|c|c|}
\hline S.no & Authors & Name of the method & $\begin{array}{l}\% \\
\text { Classification } \\
\text { Rate }\end{array}$ & $\begin{array}{l}\text { Category of age } \\
\text { classification }\end{array}$ \\
\hline 1 & Proposed method & Shape patterns on -CPFM & 98 & Child and Adulthood \\
\hline 2 & $\begin{array}{l}\text { P Chandra Sekhar } \\
\text { Reddy et.al.[18] }\end{array}$ & Shape features on IT-LBP & 95 & Child and Adulthood \\
\hline 4 & $\begin{array}{l}\text { Chandra } \quad \text { Mohan } \\
\text { et.al.[19] }\end{array}$ & $\begin{array}{l}\text { Geometrical features for } \\
\text { Child and Adulthood } \\
\text { Classification }\end{array}$ & 94.5 & Child and Adulthood \\
\hline 3 & $\begin{array}{l}\text { Young H. } \\
\text { Kwon et.al.[3] }\end{array}$ & $\begin{array}{l}\text { Age classification from facial } \\
\text { images }\end{array}$ & 78 & $\begin{array}{l}\text { Babies, adults, and } \\
\text { Senior adults. }\end{array}$ \\
\hline 4 & $\begin{array}{l}\text { Tsuneo } \\
\text { Kanno } \\
\text { et al.[20] }\end{array}$ & $\begin{array}{l}\text { Neural networks for } \text { Age } \\
\text { groups of } \quad \text { young male } \\
\text { faces. }\end{array}$ & 80 & $\begin{array}{l}\text { young males are } \\
\text { classified into age } \\
\text { groups } 12,15,18 \text { and } \\
22 \text { years }\end{array}$ \\
\hline 5 & $\begin{array}{l}\text { Wen-Bing } \\
\text { Horng } \\
\text { Cheng et.al. } \\
\text { [4] }\end{array}$ & $\begin{array}{l}\text { Facial features for } \\
\text { classification into age groups. }\end{array}$ & 90.52 & $\begin{array}{l}\text { Babies, young adults, } \\
\text { middle-aged adults, } \\
\text { and old adults }\end{array}$ \\
\hline
\end{tabular}

\section{CONCLUSION}

The present paper evaluated shape patterns on a $3 \times 3$ mask using CPFM. This CPFM forms texels from the facial image. The age variation is identified with frequency occurrences of LTMP, UTMP, and SSP patterns. This is a new approach for identifying variation of wrinkles in facial images of age varying people with shape patterns. So classification algorithm algorithm1 uses only these three shape pattern features. The percentage of classification rate is $98 \%$ and this is highest classification rate from table2. The proposed method for age classification is easy to implement and also efficient compared to other schemes. The CPFM with new shape patterns and textural properties can be extended in future work.

\section{REFERENCES}

[1] Iga, R., Izumi, K., Hayashi, H., Fukano, G., Ohtani, T.: A gender and age estimation system from face images. SICE Annual Conference in Fukui (2003) ,202-209.

[2] Lanitis, A.: On the significance of different facial parts for automatic age estimation. $14^{\text {th }}$ International Conference on Digital Signal Processing 2 (2002) 1027-1030. 
[3] Kwon, Y., Lobo, N.: Age classification from facial images. Computer Vision and Image Understanding 74(1) (1999) 1-21.

[4] Horng, W., Lee, C., Chen, C.: Classification of age groups based on facial features. Tamkang Journal of Science and Engineering 4(3) (2001) 183-192.

[5] Young-Hwan Choi , Kyungrok Kim, and Eenjun Hwang , "classification based skin aging analysis”, International Asia-Pacific Web Conference, 2010, pp. 347-349.

[6] Yun, F., Ye, X. and Huang,2007, Estimating Human Age by Manifold Analysis of Face Pictures and Regression on Aging Features, Proc. of 2007 IEEE International Conference on Multimedia and Expo, pp. 1383-1386.

[7] Rama Nathan and Chellappa R.,2006, Modeling age progression in young faces, proc. IEEE conf CVPR, vol.1, 387-394.

[8] Xin Geng, Zhi-Hua Zhou, Yu Zhang, Gang Li, Honghua Dai,2006, Learning from facial aging patterns for automatic age estimation. Proceedings of the 14th annual ACM international conference on Multimedia,307-316.

[9] Lanitis, A. , Draganova, and Christodoulou, 2004, Comparing different classifiers for automatic age estimation. IEEE Transactions on Systems, Man, and Cybernetics, Part B, 34(1), 621-628.

[10] Fu Y., Huang, T.S,2008, Human age estimation with regression on the discriminative aging manifold, IEEE Tran multimedia, vol 10,4, 578-584.

[11] Lanitis, A. ,C Taylor, and Cootes ,2002, Towards automatic simulation of aging effects on facial images , IEEE Trans. Pattern Anal.macine intelligence Vol 24,4, 442-455.

[12] Yan,S., Wang, T.S, Huang and Tang X.,2007, Ranking with uncertain labels , IEEE International Conference on Multimedia and Expo,96-99,2007.

[13] Mina Hashemian, Hossein Pourghassem,2013, Facial Emotion Processing in Autism Spectrum Disorder Based on Spectral Features of EEG Signals, International Journal of Imaging and Robotics, Volume 11, Issue Number 3, 68-80.

[14] Juha Ylioinas, Abdenour Hadid, Matti Pietikäinen, 2012, Age Classification In Unconstrained Conditions Using LBP Variants, proc. ICPR 2012, 1257-1260.

[15] Vijaya Kumar V., Jangala Sasi Kiran and Gorti Satyanarayana Murty,2013, Pattern Based Dimensionality Reduction Model For Age Classification, International Journal Of Computer Applications, Volume 79 - No 13,14-20.

[16] Jangala Sasi Kiran, Vijaya Kumar, V. and Eswara Reddy,B.,2013, Age Classifications Based on second Order Image Compressed and Fuzzy Reduced Grey Level (SICFRG) Model, International Journal On Computer Science And Engineering (IJCSE), Vol. 5 No. 06, 481-492.

[17] P.Chandra Sekhar Reddy, B.Eswara Reddy and V.Vijaya Kumar, "New Method for Classification of Age Groups Based on Texture Shape Features," International Journal of Imaging and Robotics(IJIR), Volume 15(1), pp.19-30, 2015.

[18] P.Chandra Sekhar Reddy, B. Eswara Reddy and V. Vijaya Kumar’Texton Based Shape Features on Local Binary Pattern for Age Classification," International Journal of Image, Graphics, and Signal Processing(IJIGSP), Volume4(7), pp.54-60, 2012.

[19] M. Chandra Mohan, V. Vijaya Kumar and B. Sujatha, "Classification of child and adult based on geometric features of the face using linear wavelets", International Journal of Signal and Image Processing, Vol. 1, No. 3, pp. 211 $-220,2010$.

[20] Tsuneo Kanno, 2001, Classification of Age Group Based on Facial Images of Young Males by Using Neural Networks, IEICE Trans. Inf \& Syst, VolE84-D, No 8. 